\title{
Cumulative live birth rates according to the number of oocytes retrieved following the "freeze-all" strategy
}

\author{
Zhonghua Zhao ${ }^{1,2}$, Hao Shi ${ }^{1,2}$, Jing Li $i^{1,2}$, Yile Zhang ${ }^{1,2}$, Caihong Chen ${ }^{1,2}$ and Yihong Guo ${ }^{1,2^{*}}$ (D)
}

\begin{abstract}
Background: In recent years, some studies have shown that there is a positive association between the number of oocytes retrieved and the cumulative live birth rate (CLBR) after fresh and frozen cycles of one oocyte retrieval. However, almost no studies have examined the association between the number of oocytes retrieved and the CLBR when using the "freeze-all" strategy. We performed this study to investigate the effects of an extreme oocyte yield during the first "freeze-all" cycle on the cumulative live birth rate among patients younger than 35 years old.

Methods: This was a retrospective cohort study performed in a university-affiliated reproductive medicine centre. Data obtained from 3276 women aged younger than 35 years who underwent their first "freeze-all" cycle (IVF/ICSI) were collected between January 2009 and December 2016. In all, 5025 frozen cycles took place during the followup period from January 2009 to December 2018. Patients were divided into five groups according to oocytes retrieved (group 1: 4-10 oocytes; group 2: 11-20 oocytes; group 3: 21-30 oocytes; group 4: 31-40 oocytes; group 5: $>40$ oocytes). The primary outcome was the cumulative live birth rate.
\end{abstract}

Results: Unadjusted results showed that the cumulative live birth rate significantly increased as the number of oocytes retrieved increased and reached up to $93.82 \%$ in cases with yields of $21-30$ oocytes $(P<0.05)$, after which it did not have a significant increase $(P>0.05)$. After adjusting for confounders, our results showed that the number of oocytes retrieved is an independent positive predictor of cumulative live birth rate when using a "freeze-all" strategy. $(P<0.001)$. In addition, the fertilization rate and the gonadotropin dose also influenced the cumulative live birth rate $(P<0.05)$.

Conclusions: Among women younger than 35 years old who underwent the "freeze-all" strategy, the number of oocytes retrieved positively correlated with the cumulative live birth rate. Taking both efficacy and safety into account, ovarian stimulation should be rational, and the upper limit of the oocyte yield should be no more than 30 .

Keywords: Cumulative live birth rate. Ovarian hyperstimulation syndrome. "Freeze-all" strategy. Oocyte number. In vitro fertilization

\section{Background}

After the advent of controlled ovarian stimulation (COS), multi-follicular stimulation cycles replaced natural monofollicular cycles in the clinical practice of assisted reproductive technology (ART) [1]. A conventional in vitro fertilization (IVF) procedure starts with COS and ends with the transfer of the best available embryo [1], while all

\footnotetext{
* Correspondence: 13613863710@163.com

${ }^{1}$ Center of Reproductive Medicine, The First Affiliated Hospital of Zhengzhou University, Zhengzhou 450052, People's Republic of China

${ }^{2}$ Henan Key Laboratory of Reproduction and Genetics, Zhengzhou 450052, People's Republic of China
}

surplus embryos of adequate quality are cryopreserved for later use [2]. However, under some circumstances, a fresh embryo transfer cannot be performed, and the entire cohort of viable embryos is cryopreserved [1]. This is known as the "freeze-all" strategy, in which all embryos are cryopreserved for future frozen-thawed embryo transfer, and this approach is increasingly being favoured [3]. There are many indications for the "freeze-all" strategy: ovarian hyperstimulation syndrome (OHSS), the asynchrony of embryo and endometrial receptivity, preimplantation genetic diagnosis (PGD), preimplantation genetic screening (PGS) 
[3], fertility preservation and premature progestin elevation [4]. OHSS, an iatrogenic complication of COS accompanied by a higher number of retrieved oocytes, accounts for a substantial proportion of these indications. A strategy comprising the use of a GnRH antagonist for pituitary downregulation, a GnRH agonist for ovulation triggering, and vitrification of all embryos can achieve an OHSS-free status for high responders [3]. In recent years, randomized controlled trials and observational studies have shown that better in vitro fertilization pregnancy outcomes and lower rates of obstetric and perinatal morbidity are achieved following the first frozen-thawed embryo transfer of the "freeze-all" strategy than the fresh embryo transfer [5-7]. Furthermore, some studies have examined the association between the number of oocytes retrieved and the cumulative live birth rate (CLBR) after one fresh and several frozen-thawed embryo transfers following one round of oocyte retrieval, and all of these studies found a positive association $[8,9]$. However, almost no study has examined the association between the number of oocytes retrieved and the CLBR when the "freeze-all" strategy is used. Therefore, we carried out the current study to investigate the relationship between the number of oocytes retrieved and the CLBR for the "freeze-all" strategy.

\section{Methods}

\section{Patients}

This retrospective study was based on the Clinical Reproductive Medicine Management System/Electronic Medical Record Cohort Database (CCRM/EMRCD) at the Reproductive Medical Center, First Affiliated Hospital of Zhengzhou University, and the Henan Province Key Laboratory for Reproduction and Genetics. A total of 3276 Chinese women younger than 35 years who underwent a first "freeze-all" strategy were collected between January 2009 and December 2016, and 5025 frozen cycles took place during the follow-up period from January 2009 to December 2018. Of 3276, 380 patients discontinued treatment without a live birth but with embryo(s) left in the first "freeze-all" strategy (we regarded them as dropouts). In our study, only the first "freeze-all" strategy (cycle) due to OHSS tendency or early OHSS, which occurs within 9 days of human chorionic gonadotropin (hCG) administration, was considered. Tubal disease included (1) history of surgery on the fallopian tubes: previous EP, salpingostomy, and tube reconstruction surgery and (2) hysterosalpingography examination: hydrosalpinx and salpingitis. Examples included unilateral or bilateral tubal occlusion, peritubal adhesion, unilateral or bilateral salpingectomy, or tubal ligation. PCOS was diagnosed by the Rotterdam criteria. We excluded donor cycles of oocytes or sperm, natural IVF/ICSI cycles, preimplantation genetic diagnosis (PGD) and preimplantation genetic screening (PGS) cycles. (Fig. 1).

\section{COS protocols}

The protocols were applied based on the day of the patient's menstrual cycle when the patient came to the hospital. For patients in the follicular phase, triptorelin depot (decapeptyl $3.75 \mathrm{mg}$; Ipsen Pharma, France) was injected intramuscularly on days $2-3$ of the menstrual cycle. Pituitary downregulation was achieved 28-42 days later $[\mathrm{FSH}$ (follicle-stimulating hormone) <4 IU/L, E2 (oestradiol) $<50 \mathrm{pg} / \mathrm{ml}$, LH (luteinizing hormone) $<4$ $\mathrm{IU} / \mathrm{L}$, endometrium thickness $<5 \mathrm{~mm}$ and diameter of the maximal follicle $\leq 5 \mathrm{~mm}$ ]. For patients in the luteal phase, triptorelin (Ferring $\mathrm{GmbH}, 0.1 \mathrm{mg}$, Germany; Ipsen Pharma Biotech, $0.1 \mathrm{mg}$, France) was injected intramuscularly during the midluteal phase, and 10 days later, the dose was decreased to $0.05 \mathrm{mg} / \mathrm{d}$ until pituitary downregulation [FSH (follicle-stimulating hormone) $<4$ $\mathrm{IU} / \mathrm{L}$, E2 (oestradiol) $<50 \mathrm{pg} / \mathrm{ml}, \mathrm{LH}$ (luteinizing hormone) $<4 \mathrm{IU} / \mathrm{L}$, endometrium thickness $<5 \mathrm{~mm}$ and diameter of the maximal follicle $\leq 5 \mathrm{~mm}$ ] was achieved. Ovarian hyperstimulation was started by FSH (the initial dose was based on patient age, BMI, antral follicle count, AFC, and other factors); the dose of FSH (Gonal-F, Serono, Puregon, Netherlands, u-FSH, Livzon) was adjusted based on follicle development and hormone levels, and human menopausal gonadotropin (HMG, 75 U/ampoule, Ferring $\mathrm{GmbH}$, Germany), was added if needed. When the maximal follicle was more than $20 \mathrm{~mm}$ and more than $2 / 3$ follicles $>16 \mathrm{~mm}$, human chorionic gonadotropin (2000 IU) (HCG, Lizhu. Ltd., Guangdong, China) and recombinant HCG $(250 \mu \mathrm{g})$ (Merck Serono, Italy) were administered to trigger oocyte maturation. Approximately $36-37 \mathrm{~h}$ after hCG was administered, oocyte retrieval was performed with transvaginal ultrasound guidance. IVF or ICSI was performed based on sperm parameters. Patients at risk of ovarian hyperstimulation syndrome (OHSS) were cancelled for embryo transfer and underwent whole-embryo cryopreservation. Luteal phase was supported with $60 \mathrm{mg}$ progesterone $(20 \mathrm{mg} /$ ampoule, Xianju Ltd., Zhejiang, China) from the day of ovum pick-up (OPU).

\section{Endometrial preparation schemes}

The endometrial preparation schemes for frozen embryo transfer (FET) in the current study were natural cycles and artificial (Oestrogen-Progesterone) cycles. For natural cycles, an ultrasound was performed on day 8-9 of the menstrual cycle to evaluate the endometrial thickness and the diameter of the dominant follicle. When the dominant follicle was $16-20 \mathrm{~mm}$, serum progesterone and LH levels were monitored to confirm the ovulation time. Thawing and transferring were performed 3 days after ovulation. Luteal phase support was sustained with intramuscular progesterone $40 \mathrm{mg}$ ( $20 \mathrm{mg} /$ ampoule, Xianju Ltd., Zhejiang, China) administered on the day of ovulation and 


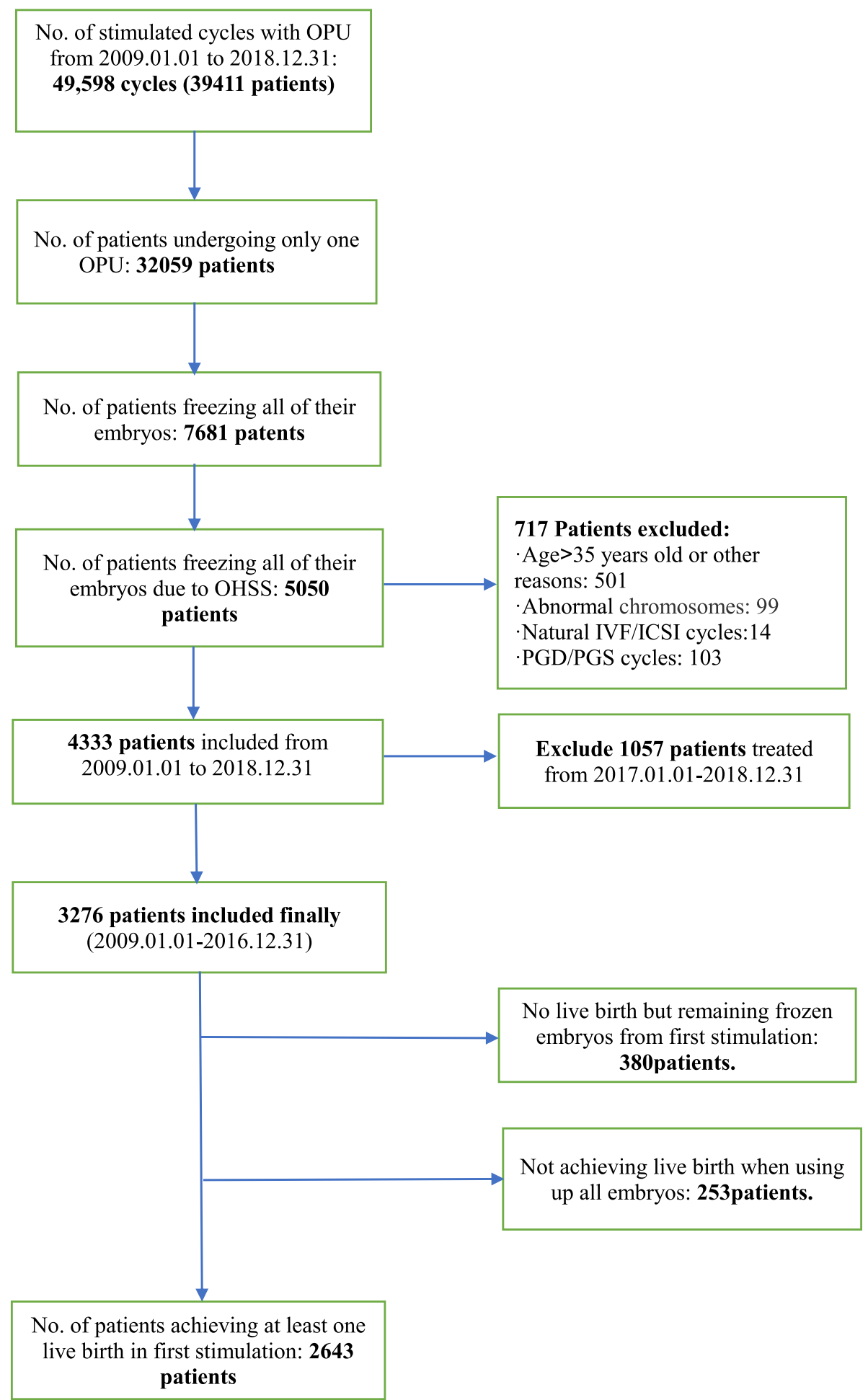

Fig. 1 Flowchart of data selection

oral dydrogesterone $20 \mathrm{mg}$ (Solvay Pharmaceuticals B.V., Veenendaal, The Netherlands) administered on the transfer day. For Oestrogen-Progesterone cycles, 2-4 mg/day of oral oestradiol ([Progynova]; Bayer, Germany) was given on day 3 of the menstrual cycle, and the dose was adjusted based on endometrial thickness. After 12-14 days, if no leading follicle was present, intramuscular progesterone $60 \mathrm{mg}$ (20 mg/ampoule, Xianju Ltd., Zhejiang, China) and 
oral dydrogesterone $10 \mathrm{mg}$ or $20 \mathrm{mg}$ (Solvay Pharmaceuticals B.V., Veenendaal, The Netherlands) were given. Embryo transfer was performed 3 days later.

\section{Outcomes}

The primary outcome was the cumulative live birth per started cycle, which was defined as at least one liveborn baby at 24 weeks of gestation resulting from an ARTinitiated cycle. Clinical pregnancy was defined as at least one gestational sac with or without foetal heart activity by ultrasound at $4-5$ weeks after embryo transfer. Live birth was defined as one or more live babies born after 24 weeks of gestation.

\section{Statistical analysis}

Statistical analyses were performed with SPSS (Statistical Package for Social Science, SPSS Inc., Chicago, IL, USA) version 19.0. Patients were classified as live birth or no live birth and were then stratified according to the number of oocytes retrieved: 4-10, 11-20, 21-30, 31-40, or $>40$ oocytes. We performed Shapiro-Wilk tests for continuous variables, and none of them fulfilled the criteria for normal distribution. Continuous variables are expressed as the medians (interquartile ranges, IQR) compared via the Mann-Whitney test (two groups) or Kruskal-Wallis test (more than two groups). Categorical variables are presented as percentages compared via the chi-square test. The differences between groups were compared using Bonferroni correction, and $P<0.05 / 10$ was set as indicating a significant difference. Multivariate logistic regression analyses were performed to explore the association between the number of retrieved oocytes and the CLBR. The results are presented as the adjusted odds ratios (aORs) with the 95\% confidence intervals (CIs). Differences were considered significant if $P<0.05$.

\section{Results}

In the current study, the median number of oocytes retrieved was 20 (interquartile range 16-24), and the distribution of aspirated oocytes is shown in Fig. 2. The median age of the women was 28 (interquartile range 25-30) years. The overall CLBR was $91.26 \%$, and the cumulative clinical pregnancy rate (CCPR) was $93.68 \%$.

\section{Baseline characteristics and treatment outcomes of women with or without live birth}

Table 1 shows that the two groups had the same baseline characteristics in terms of age, BMI, basal FSH, basal $\mathrm{LH}$, diagnosis of infertility, insemination method, stimulation protocols, and peak E2 on HCG day $(P>$ $0.05)$. The women who achieved a live birth had a significantly higher basal AFC and significantly higher fertilization rates and numbers of oocytes retrieved than were found in those without a live birth $(P<0.05)$.

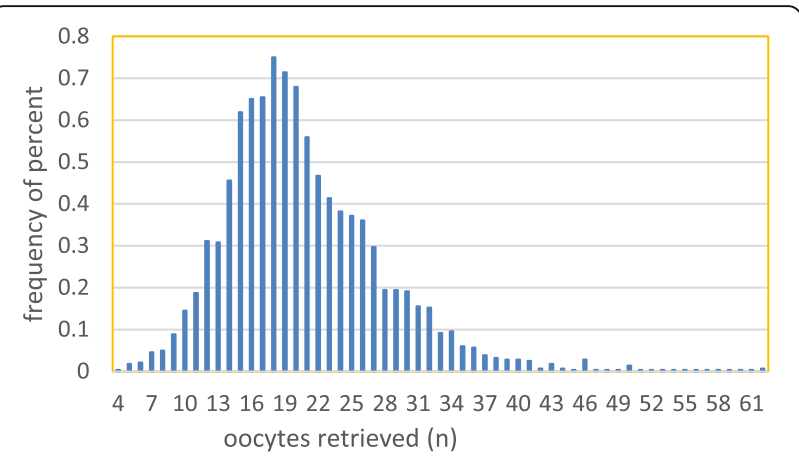

Fig. 2 Distribution of oocyte yield

However, we found that women who achieved a live birth required a lower gonadotropin dose than was used in women without live a birth $(P<0.05)$.

\section{IVF/ICSI outcome of each oocyte-stratified group in the "freeze-all" strategy analysis}

Table 2 shows that there was no significant difference in age among the five groups $(P=0.507)$. However, groups 1 and 2 had significantly higher basal FSH levels and fertilization rates than were found in groups 3,4 , and 5 . Groups 3, 4, and 5 had significantly higher peak E2 on HCG day, basal AFC and BMI than were found in groups 1 and 2. Group 2 had significantly lower basal LH levels and gonadotropin dosages than were found in group 4. The results of the Bonferroni pairwise comparison showed that the CLBR of group 1 was significantly lower than those of groups $2,3,4$, and $5(P<0.001)$ and especially group $2(72.48 \%$ vs. $90.28 \%)$. Additionally, the CLBR was significantly lower in group 2 than in group 3 (90.28\% vs. $93.82 \%, P<0.005)$. However, no significant difference was found in the CLBR among group 3, 4, and 5 (93.82\% vs. $95.26 \%$ vs. $97.22 \%, P>0.005)$. We obtained the same results when we analysed the CCPR.

\section{Multivariate logistic regression analysis of the CLBR for the "freeze-all" strategy}

Table 3 shows the multilevel logistic regression results for the number of oocytes retrieved and the CLBR. Group 1 (4-10 oocytes) was used as a reference. After adjusting for female age, BMI, basal AFC, FSH, LH levels, gonadotropin dose, peak E2 on HCG day, stimulation protocols, insemination method, and fertilization rate, the number of oocytes retrieved remained an independent positive predictor $(P<0.001)$ of CLBR. The aORs and 95\% CIs for the CLBR increased from 3.987 (2.411$6.564)$ in group 2 to $6.851(3.924-11.960)$ in group 3 , $9.579(4.122-22.259)$ in group 4 and 18.378 (2.312$146.101)$ in group 5 . The fertilization rate positively affected the CLBR, while the gonadotropin dose negatively affected the CLBR $(P<0.05)$. 
Table 1 Baseline characteristics and treatment outcomes of women with or without live birth

\begin{tabular}{|c|c|c|c|}
\hline Variables & Live birth $(N=2643)$ & No live birth $(N=253)$ & $P$ \\
\hline Age (years) & $28.0(25-30)$ & $27.5(25-31)$ & NS \\
\hline Body mass index $\left(\mathrm{kg} / \mathrm{m}^{2}\right)$ & $21.9(20.0-24.2)$ & $22.0(19.9-24.4)$ & NS \\
\hline Basal follicle-stimulating hormone (IU/L) & 5.97(5.09-7.0) & $6.11(5.01-7.15)$ & NS \\
\hline Basal luteinizing hormone (IU/L) & $5.69(4.25-8.16)$ & $5.50(4.21-7.60)$ & NS \\
\hline Basal antral follicular count (n) & $18(13-24)$ & $17(11-24)$ & 0.031 \\
\hline Cause of infertility & & & NS \\
\hline Male factor & $26.0 \%(686 / 2643)$ & $25.3 \%(64 / 253)$ & \\
\hline Tubal disease & $39.0 \%(1032 / 2643)$ & $41.5 \%(105 / 253)$ & \\
\hline PCOS & $13.7 \%(363 / 2643)$ & $9.9 \%(25 / 253)$ & \\
\hline Endometriosis & $1.3 \%(33 / 2643)$ & $0.8 \%(2 / 2530)$ & \\
\hline Unexplained & $20.0 \%(529 / 2643)$ & $22.5 \%(57 / 253)$ & \\
\hline Insemination method & & & NS \\
\hline IVF & $69.7 \%(1838 / 2643)$ & $67.6 \%(171 / 253)$ & \\
\hline ICSI & $30.3 \%(798 / 2643)$ & $32.4 \%(82 / 253)$ & \\
\hline Stimulation protocols & & & NS \\
\hline Long-acting GnRH-a long protocol & $35.4 \%(936 / 2643)$ & $33.2 \%(84 / 253)$ & \\
\hline Short-acting GnRH-a long protocol & $63.5 \%(1647 / 2643)$ & $65.2 \%(165 / 253)$ & \\
\hline Unrecorded & $1.1 \%(33 / 2643)$ & $1.6 \%(4 / 253)$ & \\
\hline Gonadotropin dosage (IU) & 1537(1237-1987) & 1650(1331-2250) & 0.002 \\
\hline Fertilization rate(\%) & $0.69(0.57-0.80)$ & $0.61(0.46-0.73)$ & 0.000 \\
\hline Peak $E_{2}$ on HCG day (IU/L) & 7276(4991-9484) & 7084(4448-9103)0 & NS \\
\hline No. of oocytes retrieved(n) & $20(16-25)$ & $18(14-21)$ & 0.000 \\
\hline
\end{tabular}

Note: Data are presented as medians (interquartile ranges, IQRs) and percentiles (numbers). $P<0.05$ was set as indicating a significant difference $P C O S$ Polycystic ovary syndrome, IVF In vitro fertilization, ICSI Intracytoplasmic sperm injection, $E_{2}$ Oestradiol, NS nonsignificant, defined as $P \geq 0.05$ The bold values indicated that the difference was significant

Table 2 IVF/ICSI outcomes for each oocyte-stratified group

\begin{tabular}{|c|c|c|c|c|c|}
\hline Variables & Group $1(N=109)$ & Group $2(N=1553)$ & Group $3(N=987)$ & Group $4(N=211)$ & Group5 $(N=36)$ \\
\hline Age (years) & $28(25-31)$ & $28(26-30)$ & $28(25-30)$ & $28(25-30)$ & $27(25-30)$ \\
\hline BMI $\left(\mathrm{kg} / \mathrm{m}^{2}\right)$ & $20.8(19.3-23.0)^{b c d}$ & $21.5(19.6-23.9)^{\alpha \beta}$ & $22.0(20.3-24.6)$ & $22.6(20.8-25.0)$ & $23.4(20.8-26.6)$ \\
\hline Basal FSH (IU/L) & $6.4(5.24-7.45)^{\mathrm{bcd}}$ & $6.1(5.22-7.14)^{\alpha \beta \gamma}$ & $5.7(4.95-6.76)$ & $5.6(4.82-6.48)$ & $5.5(4.63-6.36)$ \\
\hline Basal LH (IU/L) & $5.57(3.98-7.22)$ & $5.53(4.18-7.78)^{\beta}$ & $5.72(4.29-8.49)$ & $6.43(4.55-9.09)$ & $6.93(4.70-8.67)$ \\
\hline Peak E2 on HCG day (IU/L) & $6358(3425-8676)^{b c d}$ & $6966(4754-9122)^{a \beta \gamma}$ & 7778(5314-9732) & 8038(5477-10,929) & $8863(5696-11,939)$ \\
\hline Basal AFC (n) & $14(10-20)^{\mathrm{bcd}}$ & $17(12-22)^{\beta \gamma}$ & $20(14-24)$ & $22(15-24)$ & $24(22.5-24)$ \\
\hline Gn dosage (IU) & 1575(1231-1925) & $1537(1237-1950)^{\beta}$ & $1575(1275-2062)$ & $1637(1312-2387)$ & $1631(1406-2493)$ \\
\hline Fertilization rate (\%) & $0.70(0.60-0.88)^{b c d}$ & $0.71(0.57-0.81)^{\alpha \beta}$ & $0.67(0.55-0.76)$ & $0.67(0.56-0.75)$ & $0.62(0.53-0.74)$ \\
\hline CCPR $[\%(n / N)]$ & $81.65(89 / 109)^{a b c d}$ & $92.85(1442 / 1553)^{a}$ & $95.74(945 / 987)$ & $95.73(202 / 211)$ & $97.22(35 / 36)$ \\
\hline CLBR $[\%(n / N)]$ & $72.48(79 / 109)^{\text {abcd }}$ & $90.28(1402 / 1553)^{a}$ & $93.82(926 / 987)$ & $95.26(201 / 211)$ & $97.22(35 / 36)$ \\
\hline
\end{tabular}

Note: Data are presented as medians (interquartile ranges, IQRs)

$B M I$ Body mass index, FSH Follicle-stimulating hormone, $L H$ Luteinizing hormone, AFC Antral follicular count, Gn gonadotropin, CCPR Cumulative clinical pregnancy rate, CLBR Cumulative live birth rate, NS nonsignificant, defined as $P \geq 0.05$

The differences between groups (Bonferroni correction, $P<0.05 / 10$ ) are indicated by superscripts:

${ }^{\mathrm{a}} P,{ }^{\mathrm{b}} P,{ }^{\mathrm{c}} P$ and ${ }^{\mathrm{d}} P$ indicate group 1 vs. groups $2,3,4$, and 5 , respectively

${ }^{a} P,{ }^{\beta} P$ and ${ }^{\gamma} P$ indicate group 2 vs. groups 3 , 4and 5 , respectively

${ }^{\dagger} P$ and ${ }^{\ddagger} P$ indicate group 3 vs. groups 4 and 5 , respectively

${ }^{*} P$ indicates group 4 vs. group 5 
Table 3 Multivariate logistic regression analysis of the CLBR for the "freeze-all" strategy

\begin{tabular}{|c|c|c|}
\hline Predictors & $\mathrm{aOR}(95 \% \mathrm{Cl})$ & $P$ \\
\hline Age & $1.007(0.963-1.054)$ & 0.749 \\
\hline Body mass index & $1.003(0.955-1.052)$ & 0.961 \\
\hline Basal antral follicular count & $1.004(0.978-1.031)$ & 0.764 \\
\hline Basal follicle-stimulating hormone & $1.029(0.951-1.113)$ & 0.474 \\
\hline Basal luteinizing hormone & $1.016(0.983-1.050)$ & 0.347 \\
\hline $\log _{10}(G n)$ dosage & $0.195(0.059-0.640)$ & 0.007 \\
\hline $\mathrm{E}_{2}$ on HCG day & $0.958(0.478-1.920)$ & 0.903 \\
\hline Stimulation protocols & & 0.108 \\
\hline Long-acting GnRH-a long protocol & 1 & \\
\hline Short-acting GnRH-a long protocol & $0.686(0.473-0.994)$ & 0.047 \\
\hline Unrecorded & $1.306(0.366-4.659)$ & 0.680 \\
\hline \multicolumn{3}{|l|}{ Insemination method } \\
\hline IVF & 1 & \\
\hline ICSI & $0.808(0.596-1.097)$ & 0.172 \\
\hline Oocyte yield & & 0.000 \\
\hline $4-10$ & 1 & \\
\hline $11-20$ & $3.987(2.411-6.564)$ & 0.000 \\
\hline $21-30$ & $6.851(3.924-11.960)$ & 0.000 \\
\hline $31-40$ & $9.579(4.122-22.259)$ & 0.000 \\
\hline$\geq 40$ & 18.378(2.312-146.101) & 0.006 \\
\hline Fertilization rate & 16.877(8.100-35.166) & 0.000 \\
\hline
\end{tabular}

Note: Data are presented as aOR $(95 \% \mathrm{Cl}) ; P<0.05$ was set as indicating a significant difference

$E_{2}$ Oestradiol, IVF In vitro fertilization, ICSI Intracytoplasmic sperm injection; $\log _{10}(\mathrm{Gn})$ dose a transformation of gonadotropin dose, $\mathrm{Cl}$ Confidence interval The bold values indicate that the differences are significant

Table 4 shows that 380 patients discontinued fertility treatment at the end of follow-up. The total dropout rate was $11.5 \%$. We analysed these patients according to the number of attempts at embryo transfer. The results showed that among the first three groups, as the number of oocytes yielded increased, the number(s) of attempted embryo transfers increased, the ratio of the number of embryos used to the number of oocytes yielded increased, and the ratio of the number of oocytes left to the number of oocytes yielded decreased.

\section{Discussion}

The present study shows that among patients younger than 35 years old, the number of oocytes retrieved was an independent positive predictor of the CLBR in patients using the "freeze-all" strategy. The chance of having a live birth was $91.26 \%$ after a completed "freeze-all" cycle.

The impact of a higher number of oocytes retrieved after ovarian stimulation has been investigated in many prior studies. Advocates of mild stimulation suggest that obtaining fewer oocytes could achieve a lower embryo aneuploidy rate and a better implantation rate $[10,11]$. In contrast, other studies have reported that the numbers of usable blastocysts and euploid embryos increased with the number of oocytes retrieved [12]. These findings may explain the positive association between the number of oocytes retrieved and the CLBR. Consistent with these findings, in our study, the fertilization rate was lower, but the CLBR and CCPR were higher in groups 3,4 , and 5 than in groups 1 and 2. Our findings also showed that groups 3, 4, and 5 had higher basal FSH, basal AFC and peak E2 levels on HCG day than were found in groups 1 and 2. Similar results were found by Fatemi et al. [9]. In our study, although the included patients either showed a tendency to have OHSS or had OHSS, we eliminated the influence of this factor by administering hydroxyethyl starch and albumin as a plasma expander for several days and cancelling the fresh embryo transfer by freezing all usable embryos. These measures can bypass the detrimental effects of supraphysiological oestradiol levels and improve endometrial receptivity in a deferred frozen-thawed embryo transfer.

To date, studies investigating the association between the number of oocytes retrieved and the CLBR for the "freezeall" strategy were scarce. However, we found ten studies to date that evaluated the association between the number of oocytes retrieved and CLBR following a conventional

Table 4 Patients who discontinued treatment with no live birth but with remaining frozen embryo(s)

\begin{tabular}{llllll}
\hline Variables & \multicolumn{5}{l}{ Number of attempt(s) } \\
\cline { 2 - 6 } & $1(N=117)$ & $2(N=151)$ & $3(N=77)$ & $4(N=26)$ & $5(N=9)$ \\
\hline Age (years) & $28(26-30)$ & $28(26-31)$ & $29(27-31)$ & $29(26-31)$ & $27(24-28)$ \\
Body mass index $\left(\mathrm{kg} / \mathrm{m}^{2}\right)$ & $22.5(20.0-25.4)$ & $22.3(20.2-25.7)$ & $22.6(20.3-25.4)$ & $24.9(21.3-26.8)$ & $24.0(20.1-26.6)$ \\
No. of oocytes yielded $(\mathrm{n})$ & $6(5-9)^{\text {ab }}$ & $8(6-11)^{a}$ & $10(8-13)$ & $13(10-18)$ & $15(11.5-16)$ \\
No. of embryos used / No. of oocytes yielded $(\%)$ & $0.33(0.25-0.50)^{a b}$ & $0.57(0.40-0.71)^{a}$ & $0.73(0.55-0.83)$ & $0.73(0.64-0.88)$ & $0.92(0.80-0.93)$ \\
No. of embryos left / No. of oocytes yielded (\%) & $0.67(0.50-0.75)^{\text {ab }}$ & $0.43(0.29-0.60)^{a}$ & $0.27(0.17-0.45)$ & $0.27(0.13-0.26)$ & $0.08(0.07-0.20)$
\end{tabular}

Note: Data are presented as medians (interquartile ranges, IQRs)

The differences between groups (Bonferroni correction, $P<0.05 / 10$ ) are indicated by the following superscripts:

${ }^{\mathrm{a}} P,{ }^{\mathrm{b}} P,{ }^{\mathrm{c}} P$ and ${ }^{\mathrm{d}} P$ indicate group 1 vs. groups $2,3,4$, and 5 , respectively

${ }^{a} P_{P}{ }^{\beta} P$ and ${ }^{\gamma} P$ indicate group 2 vs. groups 3,4 and 5 , respectively

${ }^{\dagger} P$ and ${ }^{\ddagger} P$ indicate group 3 vs. groups 4 and 5 , respectively

${ }^{*} P$ indicates group 4 vs. group 5 
in vitro fertilization strategy, which consists of a single fresh and all frozen-thawed embryo transfers after one aspiration, and all found a positive correlation between the number of oocytes retrieved and the CLBR [8, 9, 12-15]. Apart from showing a positive correlation, some of these ten studies determined what oocyte number was ideal for the optimal CLBR. Magnusson et al. suggested that retrieving 18 oocytes would be optimal for cumulative live birth in patients of all ages [16]. Without mentioning age, Chen et al. from China indicated that when the oocyte number was more than 10 for PCOS patients, the CLBR did not increase [17]. A paper published in Human Reproduction showed that the ideal number of retrieved oocytes was approximately 25 for the optimal CLBR among women aged 18-35 years, while retrieving more than 30 oocytes was optimal in women aged 36-44 years, and retrieving 9 oocytes was ideal for women older than 45 years [18]. Although there was a great deal of heterogeneity in parameters such as sample size, patient age, study design, stimulation protocols et al. among these ten studies, they convinced us that there is a strong positive correlation between the number of oocytes retrieved and the CLBR in conventional in vitro fertilization strategies. However, none of these ten studies analysed the CLBR for "freeze-all" strategy. On the basis of the ten previous studies, our study further enriches the field and confirms that the same pattern exists for the "freeze-all" strategy: unadjusted results showed that the CLBR significantly increased as the number of oocytes retrieved increased and reached as high as $93.8 \%$ when $21-30$ oocytes were aspirated and then did not have a significant increase. Multivariate logistic regression revealed that the number of oocytes retrieved remained an independent predictive factor for CLBR. Our findings are supported by a few studies investigating the "freeze-all" strategy. A prospective cohort observational study showed that in the "freeze-all" cycle, a higher number of frozen blastocysts was associated with a higher CLBR in women younger than 40 years old with a normal/high response and that the highest CLBR, $88 \%$, occurred when more than 7 blastocysts were frozen [19]. However, that study was limited by its small sample size of 254 patients. Another retrospective cohort study showed that the CLBR improved as the number of oocytes yielded increased up to 25 oocytes when using the "freeze-all" strategy [20]. However, the author did not clarity the reason why all the embryos were frozen in his study. Freezing all embryos due to fertility preservation and due to OHSS are two completely different situations. Our study eliminated this heterogeneity by including the OHSS-only patients and showed that the upper limit for the number of oocytes retrieved should be no higher than 30 when using the "freeze-all" strategy. Our study also shows that the fertilization rate positively affected while the gonadotropin dosage negatively affected the CLBR. These findings are consistent with previous studies [13,21].
The dropout rate in our study was $11.5 \%$. Among the patients who discontinued, we found that as the number of oocytes increased, the attempted number of embryo transfers increased, the ratio of the number of embryos used to the number of oocytes yielded increased, and the ratio of the number of oocytes left to the number of oocytes yielded decreased. These findings were consistent with a previous study in which patients in whom fewer oocytes were retrieved were more likely to discontinue further treatment due to the couple's perception of their poor treatment prognosis and the psychological burden [22].

Given that the patients in our study were younger than 35 years old, we cannot provide any guidance for patients of advanced age. Although we tried our best to reduce selection and confounding biases, there are still inherent biases related to the retrospective design of this study; these include changes in clinical practice that occurred during the long study period. Finally, the number of patients in whom more than 40 oocytes were retrieved was small $(N=36)$. However, this still represents the real number of oocytes retrieved, as a yield of more than 40 oocytes is rare in the real world. Despite the above limitations, we believe that our study has significant implications for clinicians and patients who must make informed decisions regarding the "freeze-all" strategy. However, we do not recommend that an extreme oocyte yield should be a goal given the risks of ovarian torsion, venous thromboembolism and arterial thrombosis, particularly because achieving an oocyte retrieval number higher than 30 is pointless when using the "freeze-all" strategy. This may be because younger women should have a sufficient number of euploid embryos to provide multiple possibilities for achieving a live birth, and the surplus embryos would therefore be wasted in these individuals [15].

\section{Conclusion}

In conclusion, when using the "freeze-all" strategy, the number of oocytes retrieved is a positive predictor of the CLBR among women less than 35 years old. Given that the CLBR significantly increased with the number of oocytes retrieved, reaching as high as $93.82 \%$ when $21-30$ oocytes were aspirated, the ovarian stimulation should be rational, and the upper limit of the oocyte yield should be no more than 30 to avoid severe iatrogenic complications.

\footnotetext{
Abbreviations

AFC: Antral follicular count; ART: Assisted reproductive technology; BMI: Body mass index; CCPR: Cumulative clinical pregnancy rate; Cl: Confidence interval; CLBR: Cumulative live birth rate; COS: Controlled ovarian stimulation; FSH: Follicle-stimulating hormone; ICSI: Intracytoplasmic sperm injection; IVF: In vitro fertilization; LH: Luteinizing hormone; OHSS: Ovarian hyperstimulation syndrome; OR: Odds ratio; PCOS: Polycystic ovary syndrome; PGD: Preimplantation genetic diagnosis; PGS: Preimplantation genetic screening
}

Acknowledgments

Not applicable. 


\section{Authors' contributions}

Designer of the study: ZHZ. Data acquisition and analysis: ZHZ, HS, JL. Draft of the manuscript and interpretation: ZHZ. Revision of the manuscript: $Y H G$, YLZ, CHC. All authors approved the final manuscript.

\section{Funding}

Our work was supported by grant 201701005 from Henan Medical Science and Technology Research Project, China.

\section{Availability of data and materials}

The datasets used in the current study are available from the corresponding author on reasonable request.

\section{Ethics approval and consent to participate}

The study was approved by the Institutional Review Board and Ethics Committee of the First Affiliated Hospital of Zhengzhou University, and written informed consent was obtained from all participants.

\section{Consent for publication}

Not applicable.

\section{Competing interests}

The authors declare that they have no competing interests.

Received: 8 October 2019 Accepted: 18 February 2020

Published online: 22 February 2020

\section{References}

1. Zacà C, Bazzocchi A, Pennetta F, Bonu MA, Coticchio G, Borini A. Cumulative live birth rate in freeze-all cycles is comparable to that of a conventional embryo transfer policy at the cleavage stage but superior at the blastocyst stage. Fertil Steril. 2018:110:703-9.

2. Maheshwari A, Pandey S, Shetty A, Hamilton M, Bhattacharya S. Obstetric and perinatal outcomes in singleton pregnancies resulting from the transfer of frozen thawed versus fresh embryos generated through in vitro fertilization treatment: a systematic review and meta-analysis. Fertil Steril. 2012;98:368-377.e1-9.

3. Blockeel C, Drakopoulos P, Santos-Ribeiro S, Polyzos NP, Tournaye H. A fresh look at the freeze-all protocol: a SWOT analysis. Hum Reprod. 2016;31:491-7.

4. Shapiro BS, Daneshmand ST, Garner FC, Aguirre M, Hudson C, Thomas S. Embryo cryopreservation rescues cycles with premature luteinization. Fertil Steril. 2010;93:636-41.

5. Chen ZJ, Shi Y, Sun Y, Zhang B, Liang X, Cao Y, Yang J, Liu J, Wei D, Weng N, Tian L, Hao C, Yang D, Zhou F, Shi J, Xu Y, Li J, Yan J, Qin Y, Zhao H, Zhang $H$, Legro RS. Fresh versus frozen embryos for infertility in the polycystic ovary syndrome. N Engl J Med. 2016;375:523-33.

6. Wei D, Liu JY, Sun Y, Shi Y, Zhang B, Liu JO, Tan J, Liang X, Cao Y, Wang Z, Qin Y, Zhao H, Zhou Y, Ren H, Hao G, Ling X, Zhao J, Zhang Y, Qi X, Zhang L, Deng X, Chen X, Zhu Y, Wang X, Tian LF, Lv Q, Ma X, Zhang H, Legro RS, Chen ZJ. Frozen versus fresh single blastocyst transfer in ovulatory women: a multicentre, randomised controlled trial. Lancet. 2019;393:1310-8.

7. Roque M. Freeze-all policy: is it time for that. J Assist Reprod Genet. 2015;32: $171-6$.

8. Drakopoulos P, Blockeel C, Stoop D, Camus M, de Vos M, Tournaye H, Polyzos NP. Conventional ovarian stimulation and single embryo transfer for IVF/ICSI. How many oocytes do we need to maximize cumulative live birth rates after utilization of all fresh and frozen embryos. Hum Reprod. 2016;31: 370-6.

9. Fatemi HM, Doody K, Griesinger G, Witjes H, Mannaerts B. High ovarian response does not jeopardize ongoing pregnancy rates and increases cumulative pregnancy rates in a GnRH-antagonist protocol. Hum Reprod. 2013:28:442-52.

10. Verberg MF, Eijkemans MJ, Macklon NS, Heijnen EM, Baart EB, Hohmann FP, Fauser BC, Broekmans FJ. The clinical significance of the retrieval of a low number of oocytes following mild ovarian stimulation for IVF: a metaanalysis. Hum Reprod Update. 2009;15:5-12.

11. Baart EB, Martini E, Eijkemans MJ, Van Opstal D, Beckers NG, Verhoeff A, Macklon NS, Fauser BC. Milder ovarian stimulation for in-vitro fertilization reduces aneuploidy in the human preimplantation embryo: a randomized controlled trial. Hum Reprod. 2007;22:980-8.
12. Vaughan DA, Leung A, Resetkova N, Ruthazer R, Penzias AS, Sakkas D, Alper MM. How many oocytes are optimal to achieve multiple live births with one stimulation cycle? The one-and-done approach. Fertil Steril. 2017;107: 397-404.e3.

13. Ji J, Liu Y, Tong XH, Luo L, Ma J, Chen Z. The optimum number of oocytes in IVF treatment: an analysis of 2455 cycles in China. Hum Reprod. 2013;28: 2728-34.

14. Devesa M, Tur R, Rodríguez I, Coroleu B, Martínez F, Polyzos NP. Cumulative live birth rates and number of oocytes retrieved in women of advanced age. A single Centre analysis including 4500 women $\geq 38$ years old. Hum Reprod. 2018;33:2010-7.

15. Polyzos NP, Drakopoulos P, Parra J, Pellicer A, Santos-Ribeiro S, Tournaye $H$, Bosch E, Garcia-Velasco J. Cumulative live birth rates according to the number of oocytes retrieved after the first ovarian stimulation for in vitro fertilization/intracytoplasmic sperm injection: a multicenter multinational analysis including 15,000 women. Fertil Steril. 2018;1 10:661-670.e1.

16. Magnusson Å, Källen K, Thurin-Kjellberg A, Bergh C. The number of oocytes retrieved during IVF: a balance between efficacy and safety. Hum Reprod. 2018:33:58-64.

17. Chen $Y H$, Wang $Q$, Zhang YN, Han X, Li DH, Zhang CL. Cumulative live birth and surplus embryo incidence after frozen-thaw cycles in PCOS: how many oocytes do we need. J Assist Reprod Genet. 2017:34:1153-9.

18. Law YJ, Zhang N, Venetis CA, Chambers GM, Harris K. The number of oocytes associated with maximum cumulative live birth rates per aspiration depends on female age: a population study of 221221 treatment cycles. Hum Reprod. 2019:34:1778-87.

19. Papanikolaou E, Chartomatsidou T, Timotheou E, Tatsi P, Katsoula E, Vlachou C, Asouchidou I, Zafeiratis O, Najdecki R. In freeze-all strategy, cumulative live birth rate (CLBR) is increasing according to the number of blastocysts formed in women $<40$ undergoing Intracytoplasmic sperm injection (ICSI). Front Endocrinol (Lausanne). 2019;10:427.

20. Zhu Q, Chen Q, Wang L, Lu X, Lyu Q, Wang Y, Kuang Y. Live birth rates in the first complete IVF cycle among 20687 women using a freeze-all strategy. Hum Reprod. 2018;33:924-9.

21. Malizia BA, Hacker MR, Penzias AS. Cumulative live-birth rates after in vitro fertilization. N Engl J Med. 2009;360:236-43.

22. Sharma $V$, Allgar $V$, Rajkhowa $M$. Factors influencing the cumulative conception rate and discontinuation of in vitro fertilization treatment for infertility. Fertil Steril. 2002;78:40-6.

\section{Publisher's Note}

Springer Nature remains neutral with regard to jurisdictional claims in published maps and institutional affiliations.

Ready to submit your research? Choose BMC and benefit from:

- fast, convenient online submission

- thorough peer review by experienced researchers in your field

- rapid publication on acceptance

- support for research data, including large and complex data types

- gold Open Access which fosters wider collaboration and increased citations

- maximum visibility for your research: over $100 \mathrm{M}$ website views per year

At $\mathrm{BMC}$, research is always in progress.

Learn more biomedcentral.com/submission 